\title{
Pattern Evolution of Self-Assembled Quantum Dots Under Biaxial Stresses
}

Yaoyu Pang, and Rui Huang

Department of Aerospace Engineering and Engineering Mechanics, University of Texas, Austin, TX, 78712

\begin{abstract}
A stressed epitaxial film can undergo surface instability. The stress field and the interface interaction have profound effects on the dynamics of surface evolution that leads to selfassembled quantum dots. In this paper, by using a nonlinear evolution equation, we investigate pattern evolution of self-assembled quantum dots under general biaxial stresses. It is found that the shape of quantum dots and their spatial ordering are strongly influenced by the relative magnitudes of the biaxial stresses. Linear perturbation analysis and nonlinear numerical simulations are conducted to elucidate the effect of stress anisotropy on the process of selfassembly that selects different patterns.
\end{abstract}

\section{INTRODUCTION}

Self-assembled nanostructures in epitaxial systems are of great interests for both theoretical understanding and practical applications. An epitaxial thin film is inherently stressed due to lattice mismatch between the film and the substrate. The competition between surface energy and strain energy drives surface instability of an initially flat film [1-3]. In addition, the interface between the film and the substrate plays an important role in the later stage of surface evolution [4]. Recently, we developed a nonlinear evolution equation [5] taking into account the effects of the second-order stress field and a nonlinear wetting potential. Numerical simulations showed that the nonlinear stress field alone induces "blow-up" instability, leading to crack-like grooving in 2D and circular pit-like morphology in 3D. With the wetting potential, the blow-up is suppressed, leading to an array of discrete islands on top of a thin wetting layer. Under an equi-biaxial stress, the system is isotropic (material anisotropy was ignored), and the model predicted self-assembly of circular islands with no spatial ordering (as shown in Figure 1).
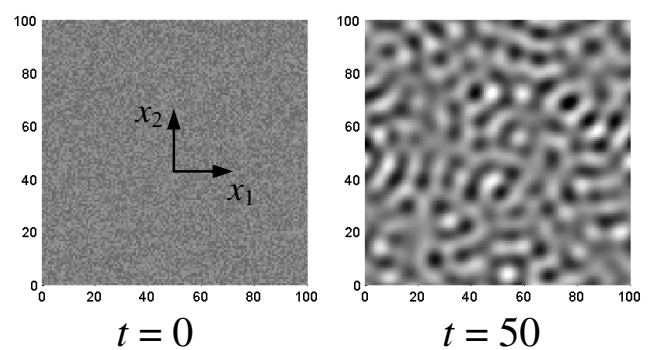
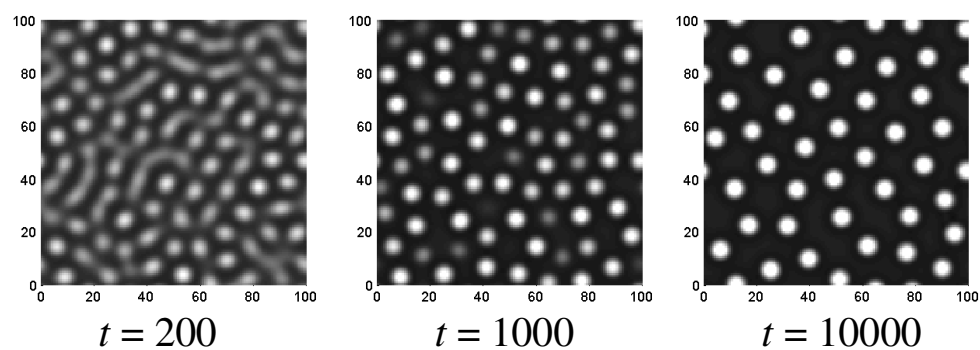

Figure 1. Evolution of surface morphology from a numerical simulation under an equi-biaxial stress $(c=1)$, starting from a random initial perturbation at $t=0$. A bright spot represents a crest of the surface. The time is normalized by a time scale $\tau$, and the length by a length scale $L$, both defined in text. 
It has been shown that material anisotropy (e.g., crystal elasticity, surface energy, and surface mobility) plays a significant role in the processes of self-organization and shape transition of epitaxial quantum dots [6,7]. On the other hand, the effect of anisotropy in the general biaxial stress state has received less attention. Recently, Berger et al. [8] and Paret [9] showed that, during a melting-crystallization process, a biaxially stressed semi-infinite solid can develop into a rich variety of patterns, especially when the applied stress is tensile in one direction and compressive in the orthogonal direction. In this paper, we investigate pattern evolution of self-assembled quantum dots under general biaxial stresses. A linear analysis and nonlinear numerical simulations are conducted to elucidate the effect of stress anisotropy on the shape of quantum dots and their spatial organization.

\section{EVOLUTION EQUATION}

In a previous study [5], a nonlinear evolution equation was derived for surface evolution of a stressed epitaxial film, which takes into account the contributions from the second-order stress field and a nonlinear wetting potential. A compact form of the equation can be written as

$$
\frac{\partial h}{\partial t}=\Omega^{2} M \frac{\partial^{2}}{\partial x_{\alpha} \partial x_{\alpha}}\left(\frac{1}{2} U_{E}^{(0)} h_{\beta} h_{\beta}+U_{E}^{(1)}+U_{E}^{(2)}-\gamma_{\beta \beta}+U_{W}\right) .
$$

where $h=h\left(x_{1}, x_{2}, t\right)$ is the evolving thickness profile of the film, $h_{\beta}=\partial h / \partial x_{\beta}$ the surface gradient, $h_{\beta \beta}=\partial^{2} h / \partial x_{\beta}^{2}$ the surface curvature to the first order, $U_{E}^{(n)}$ the $n$ th-order elastic strain energy density at the surface, $\gamma$ the surface energy density, $U_{W}$ the wetting potential, $\Omega$ the atomic volume, and $M$ the atomic mobility at the surface. A repeated Greek subscript implies summation over 1 and 2 for the in-plane coordinates $x_{1}$ and $x_{2}$.

At the reference state, the film surface is flat (i.e., $h=h_{0}$ ), and the stress is uniform. Under a general biaxial stress $\left(\sigma_{11}^{(0)}=\sigma_{1}, \sigma_{22}^{(0)}=\sigma_{2}\right.$, and $\left.\sigma_{12}^{(0)}=0\right)$, the strain energy density is

$$
U_{E}^{(0)}=\frac{1}{2 E_{f}}\left(\sigma_{1}^{2}+\sigma_{2}^{2}-2 v_{f} \sigma_{1} \sigma_{2}\right)
$$

where $E_{f}$ is Young's modulus of the film and $v_{f}$ Poisson's ratio. The material of the film is assumed to be isotropic.

In our previous study [5], the first and second-order strain energy densities were obtained via an asymptotic approach for equi-biaxial stresses (i.e., $\sigma_{1}=\sigma_{2}$ ). Under general biaxial stresses, the results are

$$
\begin{aligned}
& U_{E}^{(1)}=\sigma_{1} \frac{\partial u_{1}^{(1)}}{\partial x_{1}}+\sigma_{2} \frac{\partial u_{2}^{(1)}}{\partial x_{2}}, \\
& U_{E}^{(2)}=\frac{1+v_{f}}{E_{f}} \sigma_{\alpha \beta}^{(0)} \sigma_{\alpha \gamma}^{(0)} h_{\beta} h_{\gamma}+\sigma_{\alpha \beta}^{(0)} \frac{\partial u_{\alpha}^{(2)}}{\partial x_{\beta}}+\frac{1}{2} \sigma_{\alpha \beta}^{(1)} \frac{\partial u_{\alpha}^{(1)}}{\partial x_{\beta}},
\end{aligned}
$$


where the first and second-order surface displacements are obtained in the form of Fourier transform:

$$
\begin{aligned}
& \hat{u}_{\alpha}^{(1)}=i k_{\gamma} C_{\alpha \beta} \sigma_{\beta \gamma}^{(0)} \hat{h}, \\
& \hat{u}_{\alpha}^{(2)}=C_{\alpha \beta} \hat{\varphi}_{\beta}+C_{\alpha 3} \hat{\phi} .
\end{aligned}
$$

In the above, $\varphi_{\beta}=\sigma_{\beta \gamma}^{(1)} h_{\gamma}, \phi=\sigma_{\alpha \beta}^{(0)} h_{\alpha} h_{\beta}$, and $C_{i j}$ is a 3-by-3 matrix accounting for the stiffness of the substrate as given in the previous study [5].

Based on a transition-layer model [10], the wetting potential takes the form

$$
U_{W}=\frac{b \Delta_{\gamma}}{\pi\left(b^{2}+h^{2}\right)}
$$

where $\Delta_{\gamma}=\gamma_{f}-\gamma_{s}$ is the change in the surface energy density over a transition layer of thickness $b$.

A two-dimensional spectral method was developed to solve the evolution equation [5]. Figure 1 shows the result from a numerical simulation, where the film is subjected to an equibiaxial stress at the reference state (i.e., $\sigma_{1}=\sigma_{2}=\sigma_{0}$ ). The length is scaled by $L=\frac{\gamma_{f} \bar{E}_{s}}{2 \sigma_{0}^{2}}$, and the time by $\tau=\frac{\gamma_{f}^{3} \bar{E}_{s}^{4}}{16 \Omega^{2} M \sigma_{0}^{8}}$, where $\bar{E}_{S}=\frac{E_{S}}{1-v_{S}^{2}}$ is the plane-strain modulus of the substrate. The parameters used in the simulation are: $\bar{E}_{f} / \bar{E}_{s}=1.1, \gamma_{s} / \gamma_{f}=1.2, h_{0}=0.1 L, b=0.001 L$, and $v_{f}=v_{s}=0.25$. As discussed before, in this case, the film breaks up into circular islands on a thin wetting layer, with no spatial ordering.

\section{SYMMETRY BREAKING DUE TO STRESS ANISOTROPY}

For a general biaxial stress, let $c=\sigma_{2} / \sigma_{1}$ as the factor of stress anisotropy. A linear analysis of the evolution equation (1) leads to

$$
\frac{\partial \hat{h}}{\partial t}=s\left(k_{1}, k_{2}\right) \hat{h}
$$

where $\hat{h}\left(k_{1}, k_{2}, t\right)$ is the Fourier transform of the thickness profile and

$$
s\left(k_{1}, k_{2}\right)=\frac{1}{k\left(1-v_{s}\right)}\left[\left(k_{1}^{2}+k_{2}^{2} c^{2}\right) k^{2}-\left(k_{1}^{2}+c k_{2}^{2}\right)^{2} v_{s}\right]-k^{4}+\frac{2 b L^{2} \Delta_{\gamma}}{\pi h_{0}^{3} \gamma_{f}} k^{2} .
$$



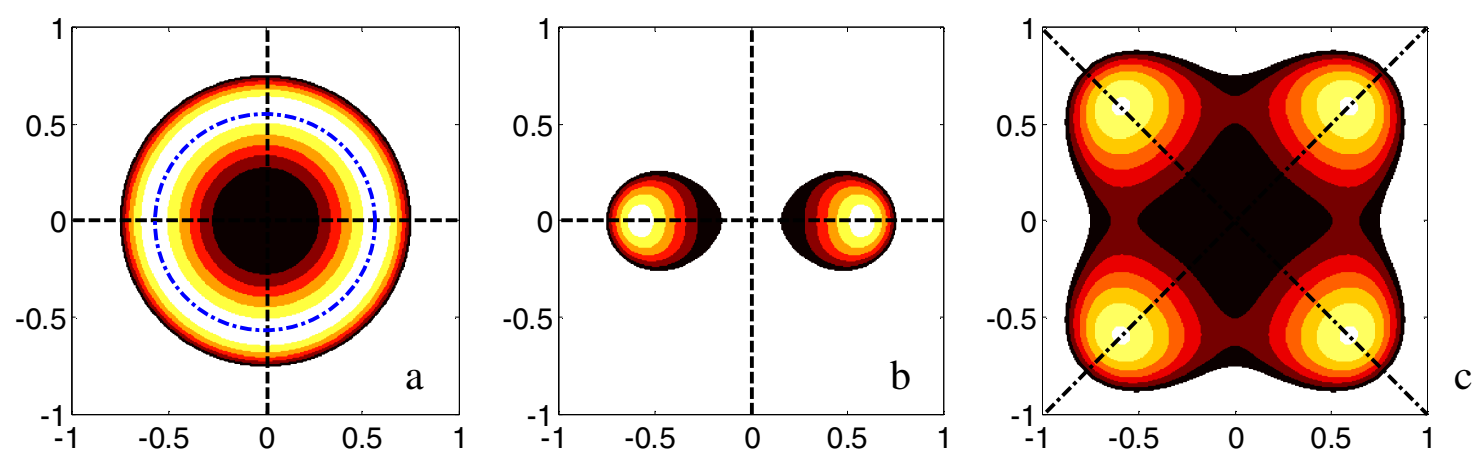

Figure 2. Contour plots of the growth rate $s\left(k_{1}, k_{2}\right)$ under different stress states. From left to right: (a) $c=1$ (equi-biaxial), (b) $c=0$ (uniaxial), and (c) $c=-1$ (pure shear).

Here the length scale $L$ and the time scale $\tau$ (with $\sigma_{1}$ in place of $\sigma_{0}$ ) are used for normalization, and $k=\sqrt{k_{1}^{2}+k_{2}^{2}}$. Therefore, in the linear regime, each Fourier component of the surface profile grows (or decays) exponentially, with the growth rate, $s$, as a function of the wave vector $\left(k_{1}, k_{2}\right)$ in the Fourier space.

Figure 2 plots the growth rate as contours in the plane of $\left(k_{1}, k_{2}\right)$. When $c=1$, the system is isotropic, and the contours are concentric circles. The growth rate is positive in an annular region (bounded by the black edges), and the fastest growing mode corresponds to a circle (dashdotted blue). The rotational symmetry leads to a chaotic pattern in the early stage of evolution and circular dots in the later stage, as shown in Figure 1.

The symmetry is broken when $c \neq 1$. As shown for $c=0$ (uniaxial stress), the fastest growing mode corresponds to two points (the white spots) located on the axis of $k_{1}$ (i.e., $k_{2}=0$ ). This suggests that the initial evolution would develop parallel striped patterns perpendicular to the direction of the uniaxial stress. In general, when $1>c>0, k_{2}=0$ for the fastest growing mode; when $c>1, k_{1}=0$. Therefore, the direction of the striped pattern depends on the relative magnitudes of the two stress components. Figure 3 shows surface evolution from a nonlinear numerical simulation for $c=0$. As predicted by the linear analysis, a parallel stripe pattern emerges at the initial stage. After a long time, however, the striped pattern coarsens and eventually breaks up into elongated islands. The aspect ratio of the island is found to be dependent upon the parameter $c$.
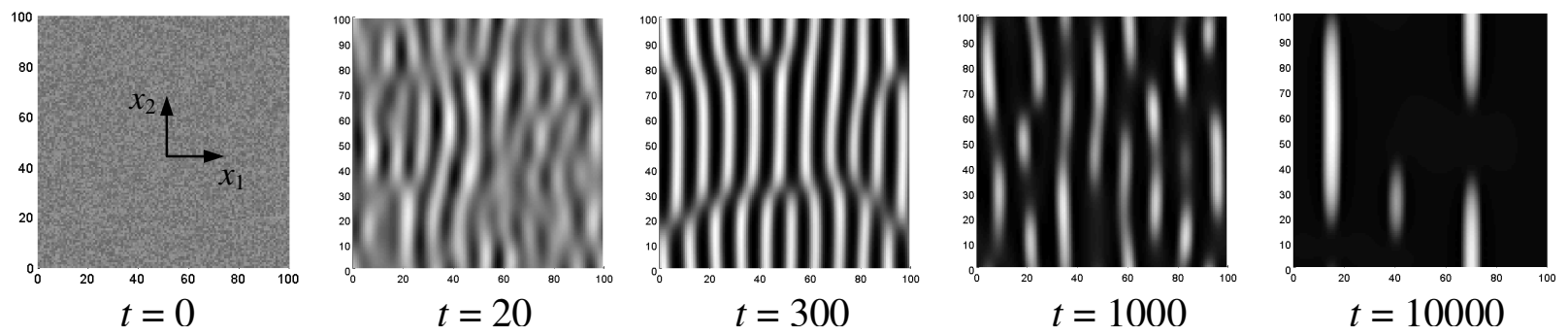

Figure 3. Evolution of surface morphology from a numerical simulation under a uniaxial stress $(c=0)$. The time is normalized by a time scale $\tau$, and the length by a length scale $L$, both defined in text. 


\section{BIFURCATION OF GROWTH MODE}

When $c<0$, a bifurcation occurs at a critical value. As shown in Figure 2(c) for $c=-1$, the fastest growing mode corresponds to four points located at an angle $\pm \pi / 4$; the two white spots in (b) split into four in (c). Define an angle $\theta$ for the wave vector so that $k_{1}=k \cos \theta$ and $k_{2}=k \sin \theta$. By setting $\partial s / \partial \theta=0$, we obtain that

$$
(c-1)\left[\left(1-v_{s}\right)(1+c)-v_{s}(1-c) \cos 2 \theta\right] \sin \theta \cos \theta=0 .
$$

Evidently, under an equi-biaxial stress $(c=1), \partial s / \partial \theta=0$ for all angles. Thus, the growth rate is independent of the angle, as shown by the circular contours in Figure 2(a). When $c \neq 1$, the growth rate depends on the angle and reaches extreme values at specific angles satisfying Eq. (10). The angle of the fastest growing mode can then be determined by examining the second derivative of the growth rate, which is plotted in Figure 4 as a function of the stress anisotropy. There exist three cases: (1) When $1>c>-\left(1-2 v_{s}\right), \sin \theta=0$ for the fastest growing mode, which gives $\theta=0$ or equivalently $\theta= \pm \pi$. (2) When $c>1$ or $c<-\left(1-2 v_{s}\right)^{-1}$, the fastest growing mode corresponds to $\cos \theta=0$, which gives $\theta= \pm \pi / 2$. Case (2) is equivalent to Case (1) by simply switching the roles of $\sigma_{1}$ and $\sigma_{2}$. (3) When $-\left(1-2 v_{s}\right)^{-1}<c<-\left(1-2 v_{s}\right)$, the angle of the fastest growing mode is given by

$$
\cos 2 \theta=\frac{(1+c)\left(1-v_{s}\right)}{(1-c) v_{s}}
$$

Consequently, a bifurcation of the fastest growing mode is predicted. When $c=-1$, the biaxial stress is equivalent to a pure shear stress, and the angle $\theta= \pm \pi / 4$.

Figure 5 shows the evolution of surface morphology from a numerical simulation with $c=-1$. As predicted by the linear analysis, the initial growth selects the fastest growing mode, in this case, with the angles $\theta= \pm \pi / 4$. A diamond type pattern emerges and ensues until the film breaks up into islands, as predicted by Berger et al. [8]. Subsequent evolution beyond the linear regime shows coarsening of the islands and eventually formation of a tilted stripe pattern. The competition of the two tilting directions leads to co-existing of long and short (broken) stripes.

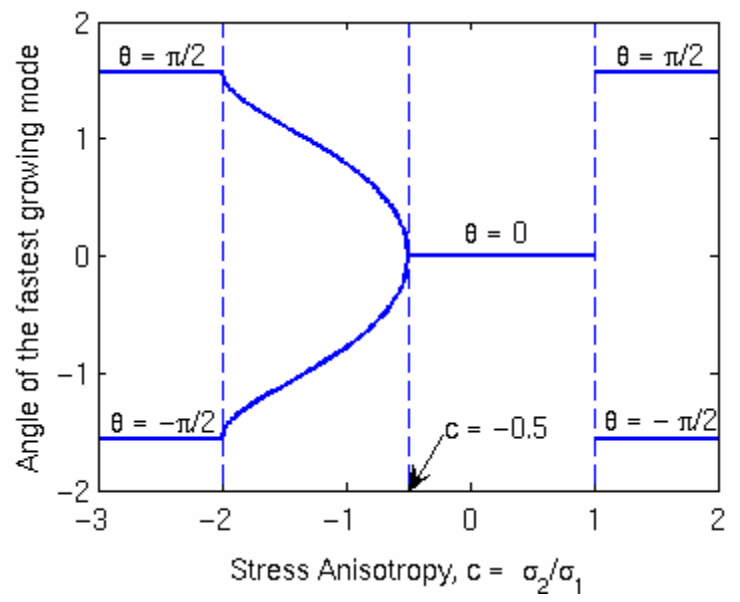

Figure 4: The angle of the fastest growing mode at the initial stage as a function of the stress anisotropy $\left(v_{s}=0.25\right)$. 

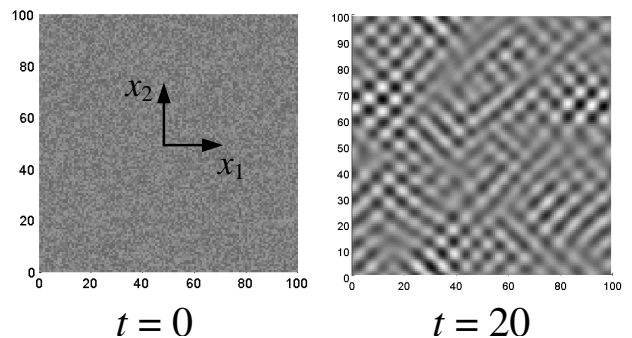

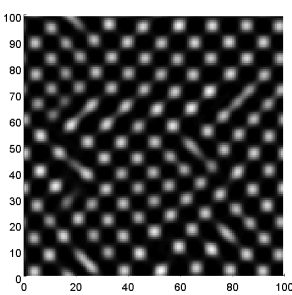

$t=200$

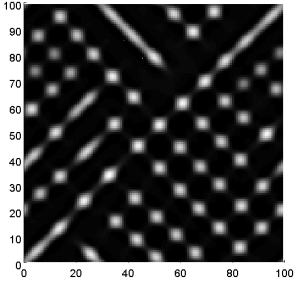

$t=1000$

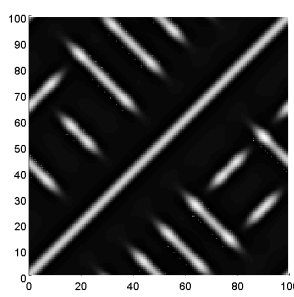

$t=10000$

Figure 5. Evolution of surface morphology from a numerical simulation with $c=-1$. The time is normalized by a time scale $\tau$, and the length by a length scale $L$, both defined in text.

\section{SUMMARY}

We have theoretically shown that, under general biaxial stresses $(c \neq 1)$, the rotational symmetry in an otherwise isotropic system is broken, leading to parallel striped patterns in the early stage of evolution and elongated islands in the late stage. Furthermore, a bifurcation of the growth mode is predicted when $-\left(1-2 v_{s}\right)^{-1}<c<-\left(1-2 v_{s}\right)$, in which case a diamond pattern and tilted stripes are predicted. This offers a rich variety of patterns for self-assembled surface structures that can be achieved by controlling the stress state. As of today, limited experimental evidences have been reported in exploring stress anisotropy (as well as other approaches of symmetry breaking) for self-assembled nanostructures [11-13]. Further studies are in progress to experimentally implement stress anisotropy (e.g., by using elastically anisotropic substrate surfaces such as $\mathrm{Si}(113)$, by patterned surface templates, or by strain engineering).

\section{ACKNOWLEDGMENTS}

The authors are grateful for the support by Department of Energy through Grant DE-FG0205ER46230.

\section{REFERENCES}

1. R.J. Asaro and W.A. Tiller, Metall. Trans. 3, 1789-1796 (1972).

2. M.A. Grinfeld, Sov. Phys. Dokl. 31, 831-834 (1986).

3. D.J. Srolovitz, Acta Metall. 37, 621-625 (1989).

4. T.V. Savina, P.W. Voorhees, and S.H. Davis, J. Appl. Phys. 96, 3127-3133 (2004).

5. Y. Pang and R. Huang, submitted (2005). Preprint available at http://www.ae.utexas.edu/ ruihuang/.

6. Y.W. Zhang, Phys. Rev. B 61, 10388-10392 (2000).

7. S.P.A. Gill, Thin Solid Films 423, 136-145 (2003).

8. P. Berger, P. Kohlert, K. Kassner, C. Misbah, Phys. Rev. Lett. 90, 176103 (2003).

9. J. Paret, Phys. Rev. E 72, 011105 (2005).

10. B.J. Spencer, Phys. Rev. B 59, $2011-2017$ (1999).

11. T. Ogino, et al., Surface Science 514, 1-9 (2002).

12. M. Hupalo and M.C. Tringides, Phys. Rev. B 73, 041405 (2006).

13. Y. Chen, et al., Appl. Phys. Lett. 76, 4004-4006 (2000). 